\title{
Spatial filters for focusing ultrasound images
}

\author{
Jensen, Jørgen Arendt; Gori, Paola
}

Published in:

2001 IEEE Ultrasonics Symposium Proceedings

Link to article, DOI:

10.1109/ULTSYM.2001.992006

Publication date:

2001

Document Version

Publisher's PDF, also known as Version of record

Link back to DTU Orbit

Citation (APA):

Jensen, J. A., \& Gori, P. (2001). Spatial filters for focusing ultrasound images. In 2001 IEEE Ultrasonics Symposium Proceedings (Vol. 2, pp. 1507-1511). IEEE. I E E E International Ultrasonics Symposium. Proceedings https://doi.org/10.1109/ULTSYM.2001.992006

\section{General rights}

Copyright and moral rights for the publications made accessible in the public portal are retained by the authors and/or other copyright owners and it is a condition of accessing publications that users recognise and abide by the legal requirements associated with these rights.

- Users may download and print one copy of any publication from the public portal for the purpose of private study or research.

- You may not further distribute the material or use it for any profit-making activity or commercial gain

- You may freely distribute the URL identifying the publication in the public portal

If you believe that this document breaches copyright please contact us providing details, and we will remove access to the work immediately and investigate your claim 


\title{
Spatial filters for focusing ultrasound images
}

\author{
Jørgen Arendt Jensen and Paola Gori \\ Ørsted•DTU, Bldg. 348, \\ Technical University of Denmark, DK-2800 Lyngby, Denmark
}

\begin{abstract}
Traditionally focusing is done by taking out one sample in the received signal from each transducer element and then sum these signals. This method does not take into account the temporal or spatial spread of the received signal from a point scatterer and does not make an optimal focus of the data. A new method for making spatial matched filter focusing of RF ultrasound data is proposed based on the spatial impulse response description of the imaging. The response from a scatterer at any given point in space relative to the transducer can be calculated, and this gives the spatial matched filter for beamforming the received RF signals from the individual transducer elements. The matched filter is applied on RF signals from individual transducer elements, thus properly taking into account the spatial spread of the received signal. The method can be applied to any transducer and can also be used for synthetic aperture imaging for single element transducers. It is evaluated using the Field II program. Data from a single $3 \mathrm{MHz}$ transducer focused at a distance of $80 \mathrm{~mm}$ is processed. Far from the transducer focal region, the processing greatly improves the image resolution: the lateral slice of the autocovariance function of the image shows a $-6 \mathrm{~dB}$ width reduction by a factor of 3.3 at $20 \mathrm{~mm}$ and by a factor of 1.8 at $30 \mathrm{~mm}$. Other simulations use a 64 elements, $3 \mathrm{MHz}$, linear array. Different receiving conditions are compared and this shows that the effect of the filter is progressively lower, but the approach always yields point spread functions better or equal to a traditional dynamically focused image. Finally, the process was applied to in-vivo clinical images of the liver and right kidney from a 28 years old male. The data was obtained with a single element transducer focused at $100 \mathrm{~mm}$. The improvement in resolution was in this case less evident and further optimization is needed.
\end{abstract}

\section{Introduction}

All current ultrasound imaging systems relies on the delaysum beamformer in which the individual received signals from multi-element transducers are delayed and summed to form a focused signal. The approach was originally devel- oped for phased array radars and was later suggested for use in ultrasound by Thurstone and von Ramm [1]. The approach is quite simple and crude, since signals are always added, never subtracted, and no filtering is employed on the individual signals.

The delay-sum beamformer assumes that the sound is received by point sources, so that the distance from the receiver to the point of interest can be calculated as the geometric distance. The time of reception is then calculated by dividing the distance by the speed of sound. This time is used for selecting the sample in the received signal that is then summed with the delayed signals from the other channels. In modern digital systems the delay is calculated dynamically for all points along the focused beam and dynamic apodization is also employed to reduce the magnitude of the side and grating lobes in the focused beam. Nevertheless, the approach does not take into account the shape of the emitted beam, the size of the receiving elements, the length of the ultrasound pulse, or that the received signals will vary as a function of spatial position relative to the receiving element and transmitting aperture. Ultrasound imaging is done in the near field of the transducer, where the ultrasound fields are very complicated. It, thus, cannot be assumed that the received signal can be modeled as a basic pulse and a time delay. Such spatial information should be incorporated into the beamforming process, and this paper suggests various methods for doing so.

The first step in devising a more accurate method for beamforming is to have a physical model for the received signal. In Section 2 the received signal is described using a model based on spatial impulse responses. This has the advantage that it encapsulates the spatial variation of the received signal for all transducer types and the extent of the transmitted field is also incorporated. Two types of more accurate processing of the received signals are then suggested in Sections 2.2, where either the peak received value is optimized by a matched filter or the resolution is optimized by employing spatially varying deconvolution filters on the individual channel data.

Simulations of the matched filter approach are shown in Section 3. It is demonstrated how the approach can be used for combining a number of measurements from a focused single element transducer to yield an image that has improved 
focusing compared to the individual measurements. Results when using a multi-element linear array are also shown. Experiments with clinical in-vivo data are shown in Section 4.

\section{Theory of filtered focusing}

The basis for making an improved focusing of ultrasound images is to have a physical understanding of the formation of the received voltage traces. The received voltage trace (RF signal) $v_{r}\left(\vec{r}_{2}, t\right)$ can be written as [2]:

$$
\begin{aligned}
v_{r}\left(\vec{r}_{2}, t\right)= & \frac{\rho_{0}}{2 c_{0}^{2}} E_{m}(t) \underset{t}{\star} \frac{\partial^{3} v(t)}{\partial t^{3}} \stackrel{\star}{t} \\
& \int_{V^{\prime}}\left[\frac{\Delta \rho\left(\vec{r}_{1}\right)}{\rho_{0}}-\frac{2 \Delta c\left(\vec{r}_{1}\right)}{c_{0}}\right] h_{p e}\left(\vec{r}_{1}, \vec{r}_{2}, t\right) d^{3} \vec{r}_{1}
\end{aligned}
$$

where $\vec{r}_{2}$ denotes the position of the transducer, $\rho_{0}$ and $c_{0}$ are the mean density and speed of sound of the medium, $E_{m}(t)$ is electro-mechanical impulse response from transducer force to voltage during reception, and $v(t)$ is the front-face velocity of the transducer during transmission. The scattering of the signal originates from the density perturbations $\Delta \rho\left(\vec{r}_{1}\right)$ and speed of sound perturbations $\Delta c\left(\vec{r}_{1}\right)$ in the tissue at the location designated by $\vec{r}_{1}$. The scattering terms are convolved with the spatial pulse-echo impulse response of the transducer designated by $h_{p e}\left(\vec{r}_{1}, \vec{r}_{2}, t\right)$, which describes how the individual point scatterers are weighted and filtered by the transducer. The received signal is constructed by a spatial convolution which sums up the contributions from all the point scatterers as shown by the last integral in the equation.

Symbolically the equation can be written as

$$
v_{r}\left(\vec{r}_{2}, t\right)=v_{p e}(t) \underset{t}{\star} f_{m}\left(\vec{r}_{1}\right) \underset{r}{\star} h_{p e}\left(\vec{r}_{1}, \vec{r}_{2}, t\right),
$$

where $\stackrel{\star}{r}$ denotes spatial convolution. $v_{p e}$ is the pulse-echo wavelet, which includes both the transducer excitation and the electro-mechanical impulse response during emission and reception of the pulse. $f_{m}$ accounts for the inhomogeneities in the tissue, and $h_{p e}$ is the pulse-echo spatial impulse response that relates the transducer geometry to the spatial extent of the scattered field. The measured signal from a point scatterer is, thus, smoothed in time by $v_{p e}(t)$ and in time and space by $h_{p e}\left(\vec{r}_{1}, \vec{r}_{2}, t\right)$, which describes the spatial extent of the point spread function. The pulse-echo spatial impulse response $h_{p e}$ consist of two terms that are convolved in time, which describe the impulse response during transmission $h_{t}\left(\vec{r}_{1}, \vec{r}_{2}, t\right)$ and reception $h_{r}\left(\vec{r}_{2}, \vec{r}_{1}, t\right)$. These can be different, which is often the case for a multi-element transducer. Here $h_{t}$ consists of a sum of spatial impulse responses for the individual elements that are amplitude scaled (apodized) and delayed to form the emit focus, and $h_{r}$ is the impulse response for a single receiving element. The equation then describes how a
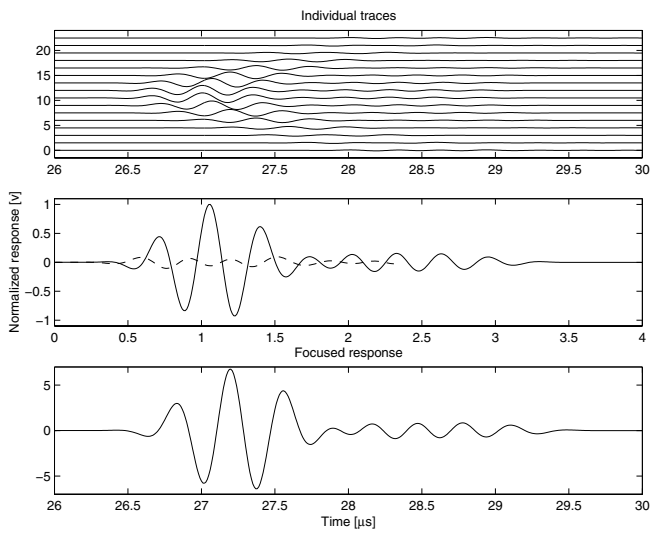

Figure 1: The top graphs shows the received signals from the individual elements of a linear array transducer with 16 elements. The received signals from the center (solid line) and last element (dashed line) of a linear array transducer with 16 elements, when they are delayed according to their distance to the point scatterer is shown in the middle graph. The focused signal is shown in the bottom graph.

single element voltage trace is constructed, and this signal is then passed through the beamformer with the other element signals to dynamically delay and thereby focus the beam in receive. An example of such responses can be seen in Fig. 1. The top graph shows the individual traces from the single elements of an array transducer with 16 elements, when the transmit focus is at $40 \mathrm{~mm}$ and the point scatterer is placed $20 \mathrm{~mm}$ from the array on its center axis. Both start time and signal shapes varies as a function of element number. A focusing at the point of interest $(20 \mathrm{~mm})$ is shown in the lower figure. The focusing significantly increases the peak amplitude of the response, but it does not eliminate the tail of the response, that is due to the variation and elongation of the response from elements furthest away from the scatterer.

From the middle graph in Fig. 1 it can also be seen that a mere alignment of the individual responses is not sufficient to add all parts of the signals in phase. The figure shows the response received on the center element and on the edge element of the array. The waveform changes as a function of relative position and the responses are therefore not in phase and a delayed response can even subtract instead of add to the focused response. This is a consequence of the spatial variation of $h_{p e}$.

Proper focusing, thus, needs to address both the distance from the elements to the scattering point through the delay and the actual shape of the response. Ideally each part of the wave should be delayed individually and summed individually to constructively add to the other element signals. This entails filtering each of the element signals with a filter that is dependent on the position of the element and on the position 
of the focus point.

\subsection{Matched filter}

One option to use on the signals is a matched filter, which optimize the peak signal amplitude to the noise power for white noise. The received signal is assumed to be given by

$$
r(t)=g(t)+n_{o}(t)
$$

where $g(t)$ is the response from the system and $n_{o}(t)$ is the noise uncorrelated with $g(t)$. A linear time-invariant filter $m(t)$ is then sought to optimize the ratio

$$
\begin{aligned}
\mathrm{SN}_{p} & =\frac{\left|y\left(t_{m}\right)\right|^{2}}{E\left\{n_{o}^{2}\left(t_{m}\right)\right\}} \\
y(t) & =g(t) * m(t),
\end{aligned}
$$

where $y\left(t_{m}\right)$ is the peak signal value at the time $t_{m}$ for the focusing. The noise is assumed stationary and the ratio can in the frequency domain be expressed as

$$
\frac{\left|y\left(t_{m}\right)\right|^{2}}{E\left\{n_{o}^{2}(t)\right\}}=\frac{\left|\int_{-\infty}^{+\infty} G(f) M(f) e^{j 2 \pi f t_{m}} d f\right|^{2}}{\int_{-\infty}^{+\infty} S_{n}(f)|M(f)|^{2} d f},
$$

where $S_{n}(f)$ is the power density spectrum of the noise. Assuming white noise and employing Schwartz' inequality gives a matched filter of

$$
M(f)=\left(G(f) e^{j 2 \pi f t_{m}}\right)^{*}=G^{*}(f) e^{-j 2 \pi f t_{m}} .
$$

The impulse response of the filter is then given by:

$$
m(t)=g\left(t_{m}-t\right) .
$$

The filter is, thus, a time reversed version of the desired signal.

The advantage of this filter is that it optimizes the peak signal to noise power, and that it, at the same time, describes how to align the received signal. The time reversal of the impulse response ensures that the resulting signal has a linear phase, and, thus, has most energy centered around the peak of the response.

\subsection{Spatial matched filter}

The signal from each channel of an array should be processed to align its output with that from the other channels. The received element signals are dependent on the element location and the scatterer's position, and a new matched filter must be used depending on the element and on the scatterer's position. The matched filter $m_{p}\left(\vec{r}_{1}, \vec{r}_{2}, t\right)$ is then given by:

$$
\begin{aligned}
m_{p}\left(\vec{r}_{1}, \vec{r}_{2}, t\right) & =p_{r}\left(\vec{r}_{1}, \vec{r}_{2},-t\right) \\
p_{r}\left(\vec{r}_{1}, \vec{r}_{2}, t\right) & =v_{p e}(t) \underset{t}{\star} h_{t}\left(\vec{r}_{1}, \vec{r}_{2}, t\right) \star{ }_{t} h_{r}\left(\vec{r}_{2}, \vec{r}_{1}, t\right),
\end{aligned}
$$
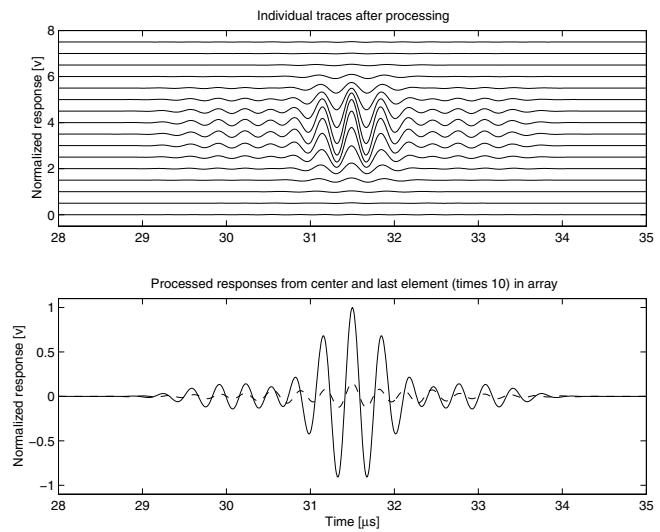

Figure 2: Processed signals from the array transducer with 16 elements.

which is dependent on the transmitter location $\vec{r}_{1}$, the receiver element location $\vec{r}_{2}$, and the electro-mechanical impulse response of the transducer $v_{p e}(t)$. The focusing is then performed by adding the processed signals from all the elements for the different locations in the image as

$$
r_{s}\left(\vec{r}_{i}\right)=\sum_{j=1}^{M} \int_{t_{i j}}^{t_{i j}+\Delta T_{i j}} v_{r}\left(\vec{r}_{j}, t\right) p_{r}\left(\vec{r}_{i}, \vec{r}_{j}, t\right) d t,
$$

where $i$ designates the point in the image, $j$ is the element number of the transducer, $t_{i j}$ is the start of the response, and $\Delta T_{i j}$ is the duration of the matched filter. The convolution integral in the equation is replaced by a correlation, since the time reversal of the response is compensated by the time reversal in the convolution.

An example of processed signals can be seen in Fig. 2, where the alignment of the main responses is clearly seen. It can also be seen how responses further away from point scatterer are severely attenuated due to the filters, which shows how apodization is automatically handled by the approach. All the responses have the characteristics of an autocorrelation function, due to the matched filter, which essentially amounts to a correlation with the assumed response (see Eq. (9)).

It should be noticed that (9) can be used for any image point, and that it is only necessary to process the point in the image that must be displayed on the screen. The approach does not put any restrictions on the transducer geometry, excitation, or impulse response. The approach can both be used for multi-element arrays and single element transducers, as long as the single element is moved compared to the scattering points during the imaging process in e.g. a polar scan. The approach improves on the focusing, if the pulse-echo spatial impulse responses are significantly different from a delta function. Normal delay focusing assumes that the geometric impulse response of the transducer is a delta function, and 

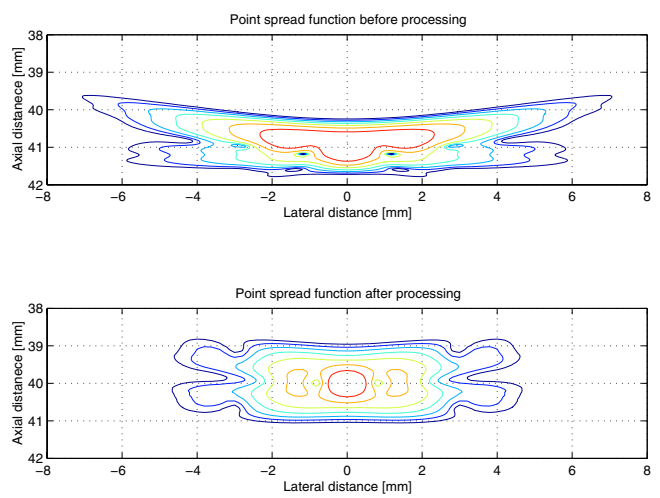

Figure 3: Contour plot ( $6 \mathrm{~dB}$ between contour) of the point spread function before (top) and after processing (bottom) for a point at a depth of $40 \mathrm{~mm}$.

that the alignment can then be done by merely delaying the responses. This is appropriate in the far-field for small element arrays and at the focus for single element transducers. The approach will, thus, work best in the near field, where long spatial impulse responses are found.

Another possibility is to use a deconvolution filter. This is done by using $p_{r}\left(\vec{r}_{1}, \vec{r}_{2}, t\right)$ in $e . g$. the design of a Wiener filter.

\section{Simulations and results}

The proposed approach for focusing has been tested through numerical simulations with the Field II program [3]. The first case analyzed is that of a single-element $3 \mathrm{MHz}$ transducer of radius $8 \mathrm{~mm}$ with geometric focus at $80 \mathrm{~mm}$. For each point in the grid, where the processing is made (identified by $\vec{r}_{i}$ in Eq. (9)), the contribution of all the lines of view are used. Eq. (9) is then applied as a sum over the lines. The number of lines used in the imaging is 100 , whereas the grid in the $x-z$ plane has $100 \times 160$ points.

Fig. 3 shows the point spread function obtained before and after processing with the method. It is seen how the response gets significantly smaller and how its gets centered exactly around $40 \mathrm{~mm}$, due to the compensation for the delay in the pulse by the matched filter.

The point spread functions have been obtained from $10 \mathrm{~mm}$ to $80 \mathrm{~mm}$ from the transducer on its axis, before and after processing. A quantification of the lateral resolution has been made by computing the 2D autocovariance of the image (envelope detected data) and considering its lateral slice [4]. The autocovariance of the envelope data is calculated after logarithmic compression to quantify the improvement in resolution. The ratio of the full widths at half maximum with and without processing is given in Fig. 4. It can be observed that in the very near field the improvement in resolution is improved significantly and that the advantage of using the

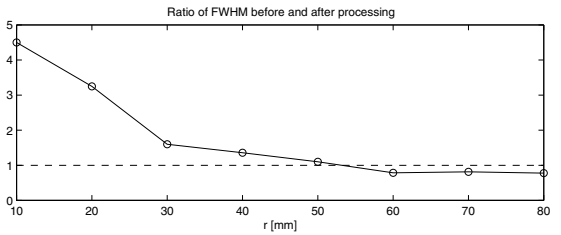

Figure 4: Ratio of full widths at half maximum of the lateral slices of the autocovariance before and after processing. Dashed line at level 1 shows the region above which the processing improves the lateral resolution

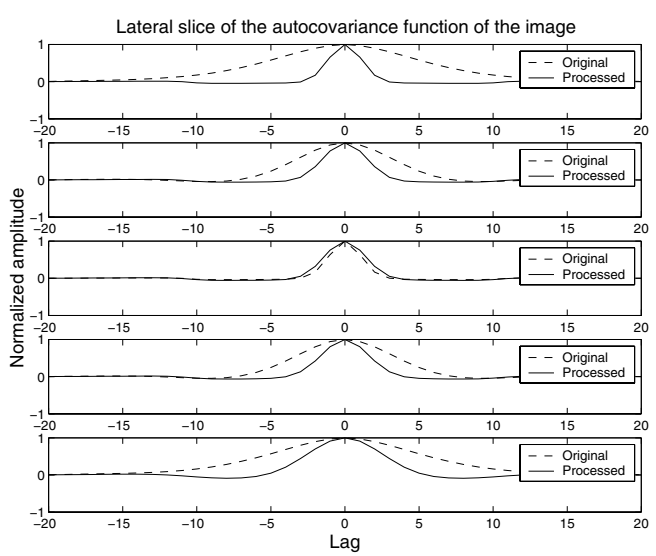

Figure 5: Lateral slice of the 2D autocovariance function of the images obtained with a linear array before (dashed line) and after (solid line) processing. A fixed focus is applied in reception.

matched filter focusing is apparent up to a distance of about $53 \mathrm{~mm}$

Another set of simulations has been devoted to the processing of the images obtained with a linear array of 128 elements (64 active) working at $3 \mathrm{MHz}$, with elements of width equal to $\lambda$ (where $\lambda$ is the wavelength) and height of $5 \mathrm{~mm}$. A phantom consists of 5 point scatterers from $20 \mathrm{~mm}$ to $60 \mathrm{~mm}$ at steps of $10 \mathrm{~mm}$ is imaged with 20 lines of view. The array is focused in transmission at $40 \mathrm{~mm}$ and a Hanning apodization is applied both in transmission and in reception. Comparisons are made by changing the reception configuration for a fixed focus at $40 \mathrm{~mm}$ and for a dynamic focusing and dynamic expanding aperture.

The resulting lateral slices of the autocovariance function for the fixed focus is shown in Figs. 5. It can be seen that the improvement in resolution obtained with the processing is large, when only a fixed focus is used in transmission. However, the effect of the filter is low or neglible when it is applied to a situation which is already very satisfactory in terms of resolution, as it is the case with dynamic focusing and aperture in reception. 

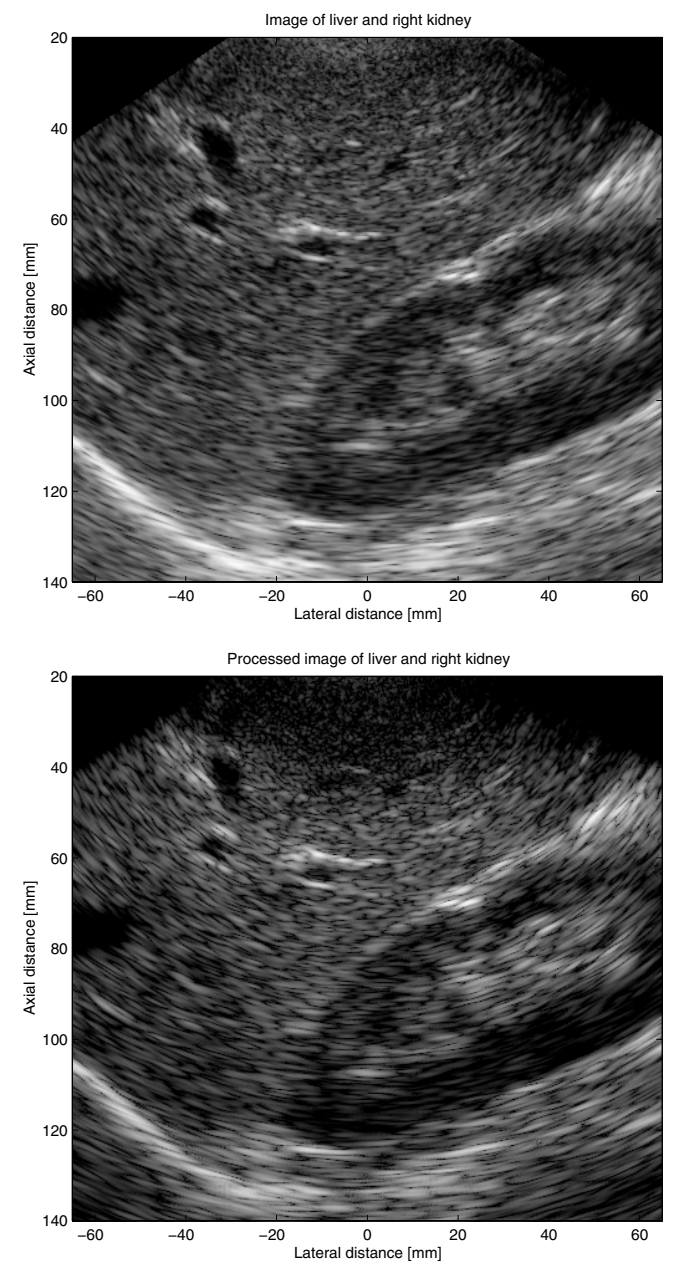

Figure 6: In-vivo images of the right kidney and liver. Top image is unprocessed and bottom image is processed by using the spatial matched filter.

\section{In-vivo images}

In-vivo images have been acquired with a $8.1 \mathrm{~mm}$ radius concave transducer focused at $100 \mathrm{~mm}$. A dedicated sampling system [5] acquiring data at $20 \mathrm{MHz}$ and 12 bits was connected to a B-K Medical 1846 scanner with a mechanically rotated transducer. The data were acquired from the liver and right kidney for a 28 years old healthy male. The data was then processed by the matched filter approach and both the unprocessed and processed data are shown in Fig. 6.

It can be seen that the difference is minute. There is a slightly better contrast in the right kidney, but the lateral resolution has not been increased. This due to the weak focusing of the transducer. Not much is gained for a weakly focused transducer, and after the focal distance there is no increase in resolution as demonstrated in Fig. 4. A more strongly focused transducer should therefore have been used in order to obtain an improvement.

\section{Conclusion}

A general approach for making focusing of ultrasound images has been suggested. The approach can take into account the spatial and temporal variations in the received signal and can through a matched filter or deconvolution filter align the responses to optimize the focusing. The approach can be employed on a set of measurement from either single or multielement transducers.

\section{Acknowledgment}

This work was supported by grant 9700883 and 9700563 from the Danish Science Foundation, by B-K Medical A/S, Denmark and by the Danish Research Academy.

\section{References}

[1] F. L. Thurstone and O. T. von Ramm. A new ultrasound imaging technique employing two-dimensional electronic beam steering. In P. S. Green, editor, Acoustical Holography, volume 5, pages 249-259, New York, 1974. Plenum Press.

[2] J. A. Jensen. A model for the propagation and scattering of ultrasound in tissue. J. Acoust. Soc. Am., 89:182-191, 1991.

[3] J. A. Jensen. Field: A program for simulating ultrasound systems. Med. Biol. Eng. Comp., 10th Nordic-Baltic Conference on Biomedical Imaging, Vol. 4, Supplement 1, Part 1:351-353, 1996.

[4] U. R. Abeyratne, A. P. Petropulu, and J. M. Reid. Higher order spectra based deconvolution of ultrasound images. IEEE Trans. Ultrason., Ferroelec., Freq. Contr., 42:1064-1075, 1995.

[5] J. A. Jensen and J. Mathorne. Sampling system for in vivo ultrasound images. In Med. Imag. V Symp., volume SPIE Vol. 1444, pages 221-231, 1991. 